\title{
Differential sphingosine-1-phosphate receptor-1 (S1PR1) protein expressions in the dorsolateral prefrontal cortex between schizophrenia Type 1 and Type 2
}

Ganesh B. Chand, ${ }^{1, a,{ }^{*}}$ Hao Jiang,,${ }^{1, a,}{ }^{*}$ Matthew Brier, ${ }^{1}$ Farzaneh Rahmani, ${ }^{1}$ Tammie L. S. Benzinger, ${ }^{1}$ C. Harker Rhodes, ${ }^{5, b}$ Zhude Tu, ${ }^{1, b}$ Dean F. Wong, ${ }^{1,2,3,4, b}$

${ }^{1}$ Mallinckrodt Institute of Radiology and Department of Radiology, ${ }^{2}$ Department of Psychiatry, ${ }^{3}$ Department of Neuroscience, ${ }^{4}$ Department of Neurology, Washington University in St. Louis School of Medicine, St. Louis, MO, USA

${ }^{5}$ NeuroDex Inc., Natick, MA, USA

aShared first authorship

${ }^{\mathrm{b}}$ Equal contribution

"Correspondence:

Ganesh B. Chand, PhD (Email: gchand@wustl.edu) or Hao Jiang, PhD (Email: haojiang@wustl.edu).

Address: 510 S. Kingshighway Boulevard, St. Louis, MO 63110

\section{ABSTRACT}

There is growing evidence that there are subtypes of schizophrenia (Bowen et al., 2019; Chand et al., 2020). Specifically, messenger ribonucleic acid (mRNA) gene expression findings on postmortem dorsolateral prefrontal cortex (DLPFC) suggest that schizophrenia patients can be divided into two groups, those with a relatively normal DLPFC transcriptome (Type 1) and those with hundreds of differentially expressed genes (Type 2). The clinical relevance of that finding is limited by the fact that autopsy tissue is required to distinguish Type 1 from Type 2 patients, however the PET target sphingosine1-phosphate receptor-1 (S1PR1) is among the genes whose mRNA expression is upregulated in Type 2 compared to Type 1 patients (Bowen et al., 2019). As a preliminary study to validate this PET target, S1PR1 protein expression was assessed by receptor autoradiography and immunohistochemistry in the DLPFC from schizophrenic patients classified as Type 1 or Type 2 based on their DLPFC transcriptomes and from controls. S1PR1 protein expression is upregulated in Type 2 compared to Type $1(p<0.05)$ supporting the possibility that positron emission tomography (PET) can be used as a clinical test to distinguish these subgroups of schizophrenic patients during life. 


\section{INTRODUCTION}

Schizophrenia is a neuropsychiatric condition that currently affects $\sim 3$ million people in the United States and $~ 7.8$ billion people worldwide (Chong et al., 2016; Cloutier et al., 2016; McCutcheon et al., 2020). Individuals with schizophrenia exhibit highly heterogeneous clinical symptoms (Derks et al., 2012), illness course (Carpenter and Kirkpatrick, 1988; Huber, 1997), and treatment response (Malhotra, 2015; Palaniyappan et al., 2013). Despite extensive efforts, understanding schizophrenia mechanism and developing targeted personalized clinical treatment are not encouraging (Insel and Cuthbert, 2015; Kapur et al., 2012). Recent findings by Bowen and colleagues (Bowen et al., 2019) suggest that the mRNA gene expression can be used to divide schizophrenic patients into two types, Type 1 schizophrenia patients with an essentially normal transcriptome in their DLPFC and Type 2 schizophrenia patients with hundreds of differentially expressed genes in their DLPFC.

A serious limitation of mRNA expression studies like that of Bowen et al (Bowen et al., 2019 ) is that they require brain tissue which is generally not available except at autopsy. Fortunately PET ligands for one of the genes differentially expressed in the Type 2 schizophrenic brains, sphingosine-1-phosphate receptor-1 (S1PR1) have been developed in the Mallinckrodt Institute of Radiology, Washington University in St. Louis (Jin et al., 2017; Liu et al., 2017; Liu et al., 2016; Liu et al., 2020; Luo et al., 2019). The present study examines the differential expression of S1PR1 in the DLPFC of Type 1 and Type 2 schizophrenic patients at the protein level as a preliminary step towards the use of PET to distinguish Type 1 from Type 2 schizophrenia during life.

S1PR1 radioligand has gained significant interest for in vivo targeted imaging of inflammation in brain diseases, with the recent FDA approval of the first orally administered S1PR1 receptor-targeted drug, FTY720 (Fingolimod) for multiple sclerosis (Marciniak et al., 2020). PET S1PR1 has recently gone into living humans successfully at Washington University in St. Louis (Liu et al., 2017; Liu et al., 2016; Liu et al., 2020; Luo et al., 2019). We have completed more than 7 PET S1PR1 studies in normal humans safely (unpublished data). Figure 1 shows examples of two healthy volunteers as part of 
initial human radiation dosimetry studies. Thus, given our ability to image S1PR1 in living human brain by PET, we are highly motivated to test our hypotheses to give further validation and justification to study S1PR1 in schizophrenia subtypes.
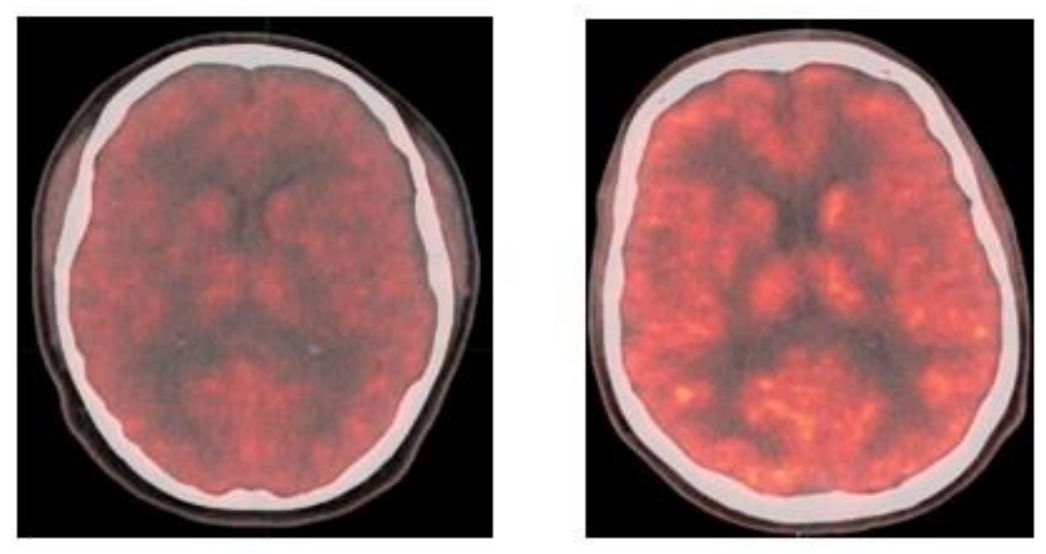

Figure 1. Examples of two healthy volunteers as part of initial human radiation dosimetry studies. They show clear passage across the blood-brain barrier and had no side effects nor adverse EKG changes.

\section{MATERIALS AND METHODS}

\section{Human brain tissues}

Human brain tissues were obtained from the Human Brain Collection Core (HBCC) at the National Institute of Mental Health (NIMH) and used in accordance with Washington University in St. Louis guidelines. All samples were stored at $-80^{\circ} \mathrm{C}$ at the Washington University Radiology labs until used.

\section{In vitro immunohistochemical staining}

In vitro immunohistochemical staining of S1PR1 was carried out in frozen sections from human DLPFC. All sections were pre-warmed at room temperature (RT) for 5 minutes and then fixed with 4\% paraformaldehyde in PBS for 10 minutes at RT, washed 3 times in PBS, and then heated in boiling water bath in antigen retrieval buffer for 30 minutes. Sections were then rinsed with PBS and blocked with $5 \%$ horse serum for 1 hour at RT. After that, all sections were stained with anti-S1PR1 antibody (Alomone, Jerusalem, Israel) overnight at $4^{\circ} \mathrm{C}$, washed and followed by incubation with ImmPRESS HRP Horse 
anti-rabbit polymer for 1 hour at RT, and developed using ImmPACT DAB (Vector Laboratories, Burlingame, CA).

\section{In vitro autoradiography analysis}

In vitro autoradiography study was carried out in frozen sections from human DLPFC using $\left[{ }^{3} \mathrm{H}\right] \mathrm{CS} 1 \mathrm{P} 1$ for S1PR1 specific protein expression. Sections were pre-incubated with HBSS buffer containing $10 \mathrm{mM}$ HEPES, $5 \mathrm{mM} \mathrm{MgCl}_{2}, 0.2 \% \mathrm{BSA}$, and $0.1 \mathrm{mM}$ EDTA at $\mathrm{pH} 7.4$ for 5 minutes at RT. All sections were then incubated with $0.5 \mathrm{nM}\left[{ }^{3} \mathrm{H}\right] \mathrm{CS} 1 \mathrm{P} 1$ for 1 hour and washed with buffer for 5 minutes at RT for three times, rinsed in ice-cold $\mathrm{H}_{2} \mathrm{O}$ and air dried overnight. Slides were incubated with Carestream BioMax autoradiography film (Carestream, Rochester, NY) in a Hypercassette autoradiography cassette (Cytiva, Amersham, UK) for 20 days along with an ART-123 Tritium Standards (American Radiolabeled Chemicals, St Louis, MO). The film was processed using a Kodak film developer (Kodak, Rochester, NY). To determine the non-specific binding, $10 \mu \mathrm{M}$ of S1PR1 specific antagonist NIBR-0213 (Cayman, Ann Arbor, MI) was introduced. The image was processed and analyzed using Fiji ImageJ.

\section{Human PET scanning}

As part of phase 1 human radiation, whole body radiation dosimetry studies have been completed in more than 7 healthy volunteers on the Siemens PET-CT Vision. High specific activity $\left[{ }^{11} \mathrm{C}\right] \mathrm{CS} 1 \mathrm{P} 1$ was synthesized at the Mallinckrodt Institute of Radiology, Washington University in St Louis and injected by IV bolus under IND FDA and Washington University School of Medicine Human Subjects (HRPO) approval. Two example human subjects representative brain PET slices are shown in Figure 1.

\section{Statistical analysis}

All data were analyzed with Prism 9.1 (GraphPad Software, San Diego, CA). One-way ANOVA followed by Fisher's LSD multiple comparison test was used to compare the tracer uptake among control, Type 1, and Type 2 schizophrenia samples. Unpaired ttest was used to compare the tracer uptake between control and schizophrenia samples. A p-value $\leq 0.05$ was considered statistically significant. 


\section{RESULTS}

\section{Human subjects}

DLPFC tissues from 20 human subjects including 10 normal controls, 5 Type 1 schizophrenia subjects, and 5 Type 2 schizophrenia subjects were used in this study.

Table 1. Human DLPFC tissues used in this study. (AA: African American; CAUC: Caucasian; Age in years).

\begin{tabular}{|cccc|cccc|ccccc|}
\hline \multicolumn{4}{|c|}{ Normal controls } & \multicolumn{4}{|c|}{ Schizophrenia Type 1 } & \multicolumn{4}{c|}{ Schizophrenia Type 2 } \\
\hline Subject & Sex & Race & Age & Subject & Sex & Race & Age & Subject & Sex & Race & Age \\
1 & M & AA & 57 & 1 & M & AA & 60 & 1 & M & AA & 63 \\
2 & M & CAUC & 63 & 2 & M & AA & 53 & 2 & F & AA & 63 \\
3 & M & AA & 60 & 3 & M & CAUC & 59 & 3 & M & CAUC & 48 \\
4 & M & CAUC & 32 & 4 & M & CAUC & 66 & 4 & M & CAUC & 39 \\
5 & M & AA & 58 & 5 & M & CAUC & 44 & 5 & M & AA & 53 \\
6 & M & CAUC & 54 & & & & & & & & \\
7 & M & CAUC & 51 & & & & & & & & \\
8 & M & CAUC & 49 & & & & & & & & \\
9 & M & AA & 64 & & & & & & & & \\
10 & F & AA & 64 & & & & & & & & \\
\hline
\end{tabular}

\section{Immunohistochemistry of S1PR1}

Immunostaining of S1PR1 was performed in control and schizophrenia samples (Figure 2). In general, S1PR1 was mainly expressed in the gray matter of the DLPFC. In particularly, the expression of S1PR1 was relatively high in outer granular layer, outer pyramidal layer, inner granular layer, inner pyramidal layer, and the multiform layer, with no to very low amount in the molecular layer of gray matter and white matter. No significant morphological difference was identified between normal control and schizophrenia subjects. 

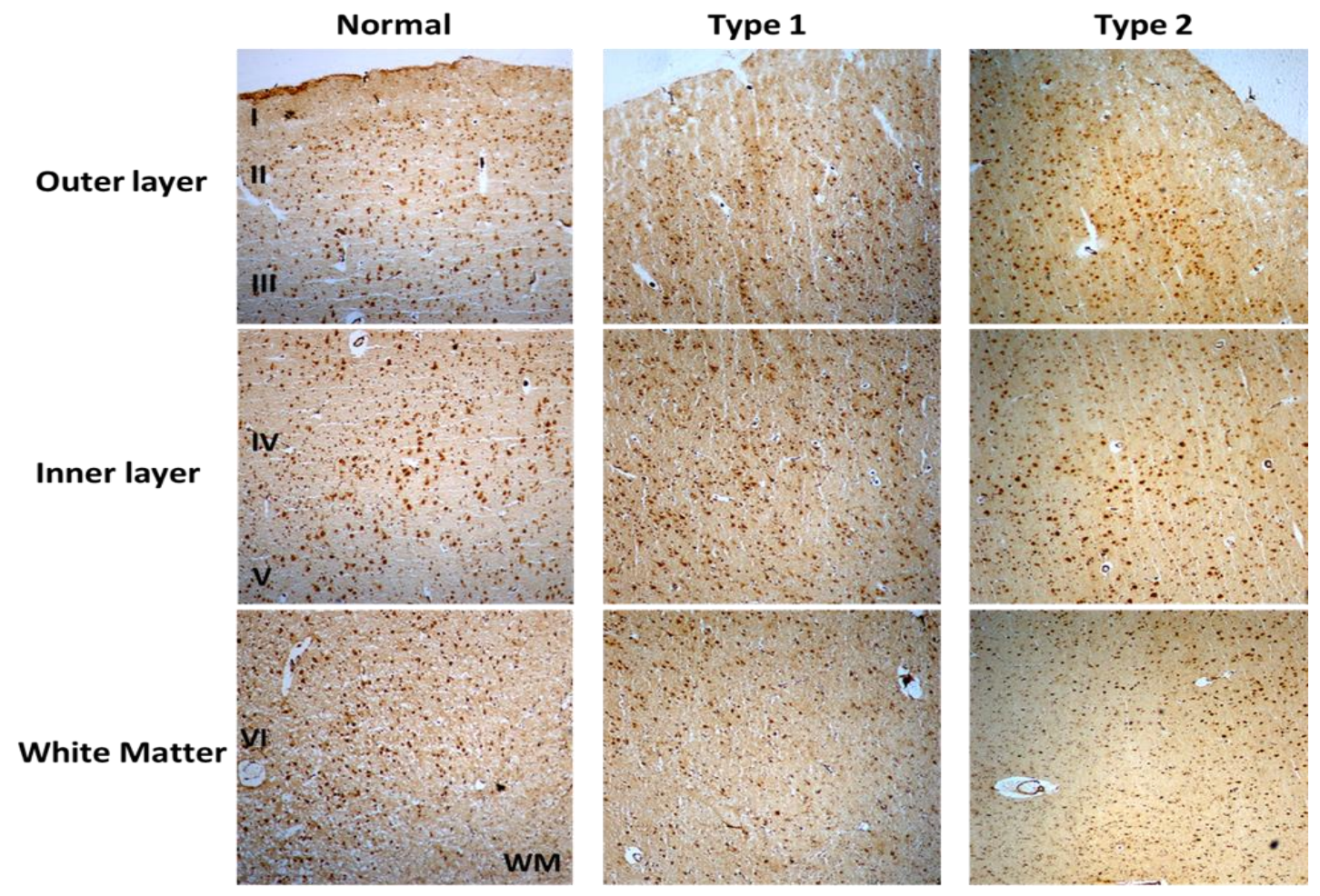

Figure 2. Immunohistochemistry of S1PR1 in control and schizophrenia DLPFC.

\section{Autoradiography of S1PR1}

Autoradiograph analysis of S1PR1 was performed in control and schizophrenia DLPFC samples (Figure 3).

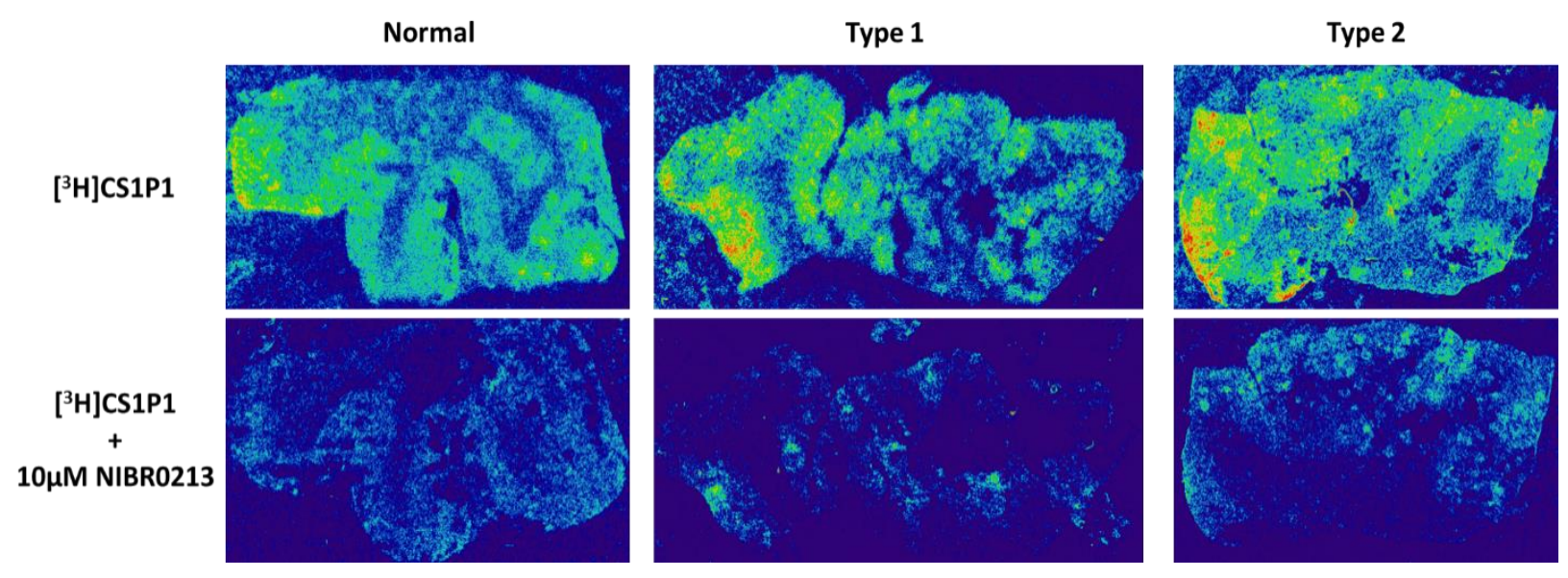

Figure 3. Representative images of autoradiograph analysis of S1PR1 using S1PR1 specific $\left[{ }^{3} \mathrm{H}\right] \mathrm{CS} 1 \mathrm{P} 1$ in control and schizophrenia DLPFC. 
The distribution pattern of $\left[{ }^{3} \mathrm{H}\right] \mathrm{CS} 1 \mathrm{P} 1$ matched well with immunostaining analysis using S1PR1 specific antibody, indicating $\left[{ }^{3} \mathrm{H}\right] \mathrm{CS} 1 \mathrm{P} 1$ is specific to S1PR1 in post mortem human tissues. Similar to S1PR1 immunostaining analysis, S1PR1 specific [ $\left.{ }^{3} \mathrm{H}\right] \mathrm{CS} 1 \mathrm{P} 1$ was mainly distributed in the gray matter of DLPFC, with no to very low amount of $\left[{ }^{3} \mathrm{H}\right] \mathrm{CS} 1 \mathrm{P} 1$ distributed in the white matter region. Comparing with immunohistochemistry analysis, autoradiography provides both quantification and localization of radioligand at the same time in distinct anatomical structures, and enable us to quantify the absolute amount of $\left[{ }^{3} \mathrm{H}\right] \mathrm{CS} 1 \mathrm{P} 1$ in control and different types of schizophrenia subjects.

Comparing with control and different types of schizophrenia samples, no significant difference was identified among different sample groups using one-way ANOVA $(F(2,13)$ $=2.795, p=0.0978$ ). Fisher's LSD test showed no significant difference between control and Type 1 or Type 2 schizophrenia samples with a $p$ value of 0.5813 and 0.0806 ; the tracer uptake in the Type 2 schizophrenia samples was significantly higher than the Type 1 schizophrenia samples with a $p$ value of 0.0480 (Figure 4A). Unpaired t-test showed no statistical difference between normal controls and all schizophrenia subjects with a $p$ value of 0.4326 even though there was a slight trend for higher means in the latter.
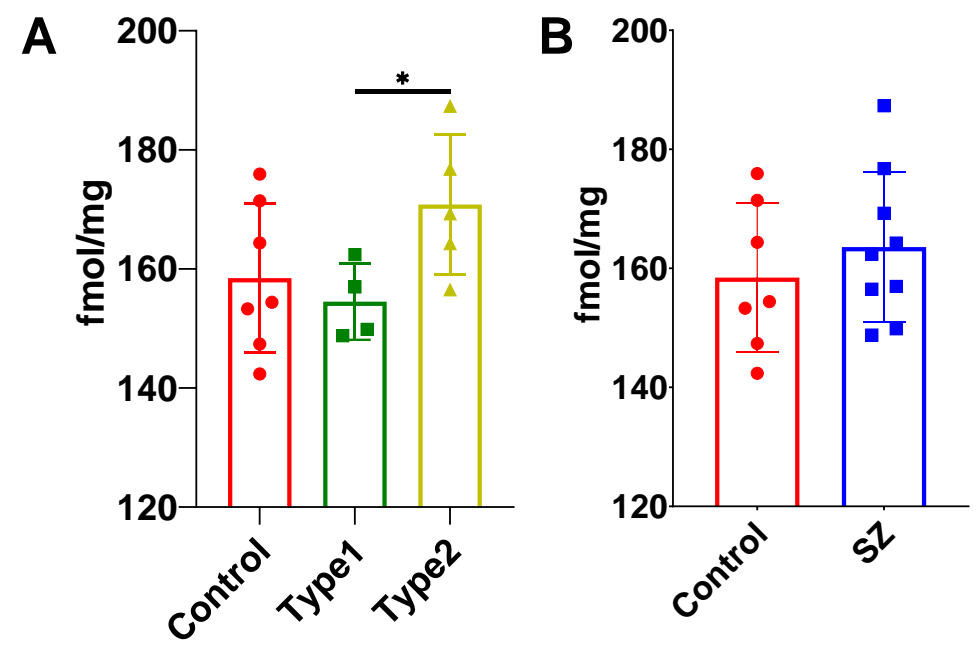

Figure 4. Autoradiograph analysis of S1PR1 using S1PR1 specific $\left[{ }^{3} \mathrm{H}\right] \mathrm{CS} 1 \mathrm{P} 1$ in control and schizophrenia DLPFC. A) Comparison among control, schizophrenia Type 1 and schizophrenia Type 2 (* represents $p<0.05$ ), and B) Comparison between control and schizophrenia (SZ) (Type 1 and Type 2 combined). 


\section{DISCUSSION}

In this study, we evaluated the expression of S1PR1 and distribution of S1PR1 specific tracer $\left[{ }^{3} \mathrm{H}\right] \mathrm{CS} 1 \mathrm{P} 1$ in human DLPFC tissues from normal control, Type 1 and Type 2 schizophrenia subjects. Our data showed, in the DLPFC of human, S1PR1 is heavily expressed in the gray matter region with no to very low amount in the white matter regions. Similar to the immunostaining study, autoradiograph analysis using $\left[{ }^{3} \mathrm{H}\right] \mathrm{CS} 1 \mathrm{P} 1$ showed a relatively high tracer uptake in the gray matter of DLPFC whereas no to very low amount of $\left[{ }^{3} \mathrm{H}\right] \mathrm{CS} 1 \mathrm{P} 1$ was identified in the white matter region. Tracer uptake in the Type 2 schizophrenia samples was significantly higher than the Type 1 schizophrenia samples.

The present findings are consistent with a previous study in other regions of human brain (Nishimura et al., 2010) that suggests S1PR1 is mainly localized in gray matter and confirmed the specificity of our S1PR1 specific radioligand $\left[{ }^{3} \mathrm{H}\right] \mathrm{CS} 1 \mathrm{P} 1$. This study extends the findings in Bowen et al (Bowen et al., 2019) by demonstrating that S1PR1 protein as well as mRNA is differentially expressed in the DLPFC of Type 2 schizophrenic patients. The trend of tracer uptake between control and all schizophrenia subjects was similar to previous findings using RT-PCR (Esaki et al., 2020). By dividing schizophrenia patients into types, it appeared that only Type 2 has significantly upregulated S1PR1.

These results have important implications for the future schizophrenia subtype-based studies with in vivo PET S1PR1. The S1PR1 has recently emerged as a promising radiotracer for in vivo PET imaging (Liu et al., 2017; Liu et al., 2016; Liu et al., 2020; Luo et al., 2019). The S1PR1 protein upregulation only in Type 2 demonstrated in this study was in line with previous reports on the S1PR1 mRNA upregulation only in Type 2 schizophrenia patients (Bowen et al., 2019). There is mounting evidence that schizophrenia has two neuroanatomical types (Chand et al., 2020). Thus, the present findings and previous reports taken together indicate that both S1PR1 protein and S1PR1 mRNA upregulate in DLPFC only in a subset of schizophrenia, and how these results relate to a recently discovered neuroanatomical types (Chand et al., 2020) remain to be explored. 
We should acknowledge the following limitations. The sample size is relatively small for this pilot study, and the future studies should focus on replicating these findings in larger samples. In addition, it remains to be investigated S1PR1 protein and S1PR1 mRNA expressions in other brain regions besides DLPFC of controls and Type 1 and Type 2 schizophrenia patients.

In conclusion, the present study evaluated DLPFC postmortem tissues from control, schizophrenia Type 1 and Type 2, demonstrated S1PR1 protein is heavily expressed in gray matter region, and most importantly showed that only Type 2 has upregulated S1PR1 protein expression in line with previous S1PR1 mRNA results. Overall, these findings suggest S1PR1 might serve as a candidate target in schizophrenia subtypes with PET where protein is the target.

\section{ACKNOWLEDGMENT}

Authors would like to acknowledge the Human Brain Collection Core (HBCC), National Institute of Mental Health (NIMH) for human DLPFC postmortem tissues, S. Marenco, MD, Acting Director of HBCC, and Mallinckrodt Institute of Radiology internal funds (DFW).

\section{REFERENCES}

Bowen, E.F.W., Burgess, J.L., Granger, R., Kleinman, J.E., Rhodes, C.H., 2019. DLPFC transcriptome defines two molecular subtypes of schizophrenia. Transl Psychiatry 9, 147.

Carpenter, W.T., Kirkpatrick, B., 1988. The heterogeneity of the long-term course of schizophrenia. Schizophr Bull 14, 645-652.

Chand, G.B., Dwyer, D.B., Erus, G., Sotiras, A., Varol, E., Srinivasan, D., Doshi, J., Pomponio, R., Pigoni, A., Dazzan, P., Kahn, R.S., Schnack, H.G., Zanetti, M.V., Meisenzahl, E., Busatto, G.F., Crespo-Facorro, B., Pantelis, C., Wood, S.J., Zhuo, C., Shinohara, R.T., Shou, H., Fan, Y., Gur, R.C., Gur, R.E., Satterthwaite, T.D., Koutsouleris, N., Wolf, D.H., Davatzikos, C., 2020. Two distinct neuroanatomical subtypes of schizophrenia revealed using machine learning. Brain 143, 1027-1038. 
Chong, H.Y., Teoh, S.L., Wu, D.B.C., Kotirum, S., Chiou, C.F., Chaiyakunapruk, N., 2016. Global economic burden of schizophrenia: a systematic review. Neuropsychiatric Disease and Treatment 12, 357-373.

Cloutier, M., Aigbogun, M.S., Guerin, A., Nitulescu, R., Ramanakumar, A.V., Kamat, S.A., Delucia, M., Duffy, R., Legacy, S.N., Henderson, C., Francois, C., Wu, E., 2016. The Economic Burden of Schizophrenia in the United States in 2013. J Clin Psychiatry 77, 764-771.

Derks, E.M., Allardyce, J., Boks, M.P., Vermunt, J.K., Hijman, R., Ophoff, R.A., Group, 2012. Kraepelin was right: a latent class analysis of symptom dimensions in patients and controls. Schizophr Bull 38, 495-505.

Esaki, K., Balan, S., Iwayama, Y., Shimamoto-Mitsuyama, C., Hirabayashi, Y., Dean, B., Yoshikawa, T., 2020. Evidence for Altered Metabolism of Sphingosine-1-Phosphate in the Corpus Callosum of Patients with Schizophrenia. Schizophr Bull 46, 11721181.

Huber, G., 1997. The heterogeneous course of schizophrenia. Schizophr Res 28, 177185.

Insel, T.R., Cuthbert, B.N., 2015. Brain disorders? Precisely. Science 348 (6234), 499500.

Jin, H., Yang, H., Liu, H., Zhang, Y., Zhang, X., Rosenberg, A.J., Liu, Y., Lapi, S.E., Tu, Z., 2017. A promising carbon-11-labeled sphingosine-1-phosphate receptor 1specific PET tracer for imaging vascular injury. J Nucl Cardiol 24, 558-570.

Kapur, S., Phillips, A.G., Insel, T.R., 2012. Why has it taken so long for biological psychiatry to develop clinical tests and what to do about it? Mol Psychiatry 17, 11741179.

Liu, H., Jin, H., Yue, X., Han, J., Baum, P., Abendschein, D.R., Tu, Z., 2017. PET Study of Sphingosine-1-Phosphate Receptor 1 Expression in Response to Vascular Inflammation in a Rat Model of Carotid Injury. Mol Imaging 16, 1536012116689770.

Liu, H., Jin, H., Yue, X., Luo, Z., Liu, C., Rosenberg, A.J., Tu, Z., 2016. PET Imaging Study of S1PR1 Expression in a Rat Model of Multiple Sclerosis. Mol Imaging Biol 18, 724-732. 
Liu, H., Luo, Z., Gu, J., Jiang, H., Joshi, S., Shoghi, K.I., Zhou, Y., Gropler, R.J., Benzinger, T.L.S., Tu, Z., 2020. In vivo Characterization of Four (18)F-Labeled S1PR1 Tracers for Neuroinflammation. Mol Imaging Biol.

Luo, Z., Gu, J., Dennett, R.C., Gaehle, G.G., Perlmutter, J.S., Chen, D.L., Benzinger, T.L.S., Tu, Z., 2019. Automated production of a sphingosine-1 phosphate receptor 1 (S1P1) PET radiopharmaceutical [(11)C]CS1P1 for human use. Appl Radiat Isot 152, 30-36.

Malhotra, A.K., 2015. Dissecting the Heterogeneity of Treatment Response in FirstEpisode Schizophrenia. Schizophr Bull 41, 1224-1226.

Marciniak, A., Camp, S.M., Garcia, J.G.N., Polt, R., 2020. In silico Docking Studies of Fingolimod and S1P1 Agonists. Front Pharmacol 11, 247.

McCutcheon, R.A., Reis Marques, T., Howes, O.D., 2020. Schizophrenia-An Overview. JAMA Psychiatry 77, 201-210.

Nishimura, H., Akiyama, T., Irei, I., Hamazaki, S., Sadahira, Y., 2010. Cellular localization of sphingosine-1-phosphate receptor 1 expression in the human central nervous system. The journal of histochemistry and cytochemistry : official journal of the Histochemistry Society 58, 847-856.

Palaniyappan, L., Marques, T.R., Taylor, H., Handley, R., Mondelli, V., Bonaccorso, S., Giordano, A., McQueen, G., DiForti, M., Simmons, A., David, A.S., Pariante, C.M., Murray, R.M., Dazzan, P., 2013. Cortical folding def ects as markers of poor treatment response in first-episode psychosis. JAMA Psychiatry 70, 1031-1040. 